\title{
An Elucidation of the Symmetry of Length Contraction Predicted by the Special Theory of Relativity
}

\author{
Koshun Suto ${ }^{1}$ \\ ${ }^{1}$ Chudaiji Buddhist Temple, Isesaki, Japan \\ Correspondence: Koshun Suto, Chudaiji Buddhist Temple, Isesaki, Japan. Tel: 81-270-23-9980. E-mail: \\ koshun_suto129@mbr.nifty.com
}

Received: April 17, 2017

Accepted: April 28, 2017

Online Published: May 31, 2017

doi:10.5539/apr.v9n3p31

URL: https://doi.org/10.5539/apr.v9n3p31

\begin{abstract}
In this paper, consider a rod A (inertial frame A) and rod B (inertial frame B) moving at constant velocity relative to each other. Assume that the lengths of two rods are equal when they are stationary. According to the STR, when length in the direction of motion of rod B, moving at constant velocity, is measured from inertial frame A, the rod contracts in the direction of motion. Also, the time which elapses on clock in inertial frame B is delayed compared to the time which elapses on clock in inertial frame A. If, conversely, inertial frame A is measured from inertial frame B, rod A contracts in the direction of motion, and the time which elapses on clock is delayed. However, according to classical common sense, if rod B contracts when measured from inertial frame A, then rod A measured from rod B must be longer than rod B. Thus, this paper discusses the symmetry of rod contraction, and elucidates this problem. It is found, based on the discussion in this paper, that the contraction of a rod includes true physical contraction, and relativistic contraction obtained due to measurement using the method indicated by Einstein. However, in the STR, any two inertial frames are equivalent, and therefore is not possible to accept points such as the fact that reasons for contraction are different. This paper concludes that STR is not a theory which describes the objective state of reality.
\end{abstract}

Keywords: Special Theory of Relativity, Classical Stationary System, Classical Moving System, Relativistic Stationary System, Length Contraction, Velocity Vector

\section{Introduction}

In the era of classical physics, as exemplified by Newtonian mechanics, it was thought that physical laws exist independently of the existence of human beings. The role of physics was to discover physical laws, and describe them in the language of mathematics.

Now, consider a rod A (inertial frame A) and rod B (inertial frame B) moving at constant velocity relative to each other. Assume that the lengths of two rods are equal when they are stationary.

According to the STR, when length in the direction of motion of rod B, moving at constant velocity, is measured from inertial frame A, the rod contracts in the direction of motion. Also, the time which elapses on clock B in inertial frame B is delayed compared to the time which elapses on clock A in inertial frame A.

If, conversely, inertial frame A is measured from inertial frame B, rod A contracts in the direction of motion, and the time which elapses on clock A is delayed.

According to Einstein's "principle of relativity," the two inertial frames are equivalent, and thus the same results are obtained no matter which inertial frame measurement is carried out from. The essence of STR is the symmetry of the theory.

However, according to classical common sense, if rod B contracts when measured from inertial frame A, then rod A measured from rod B must be longer than rod B.

The author has already discussed the symmetry of time delay in another paper (Suto, 2016-2017): The symmetry of length contraction is derived quantitatively from the formula for Lorentz transformations. However, the STR does not explain the reason why the rod contracts. Thus, this paper discusses the symmetry of rod contraction, and elucidates this problem. 
Sections 2 to 5 are preparatory stages for section 6 . Section 2 discusses the "principle of constancy of light speed" adopted by Einstein. Section 3 explains the method of discriminating between a "classical stationary system" and "classical moving system." Sections 4 and 5 discuss the contraction which can be predicted from a classical perspective. Here, the term "classical" is used when discussing objective reality which exists regardless of the observer. Section 6 employs the method of clock synchronization proposed by Einstein. It also elucidates the symmetry of rod contraction by applying the principle of relativity.

\section{The "Principle of Constancy of Light Speed E" Introduced by Einstein}

When Einstein developed the STR, he assumed the "principle of relativity" and the "principle of constancy of light speed." The latter includes the following two principles.

"Any ray of light moves in the "stationary" system of coordinates with the determined velocity $c$, whether the ray be emitted by a stationary or by a moving body." (Einstein, 1923):

"Let a ray of light start at the "A time" $t_{\mathrm{A}}$ from A towards B, let it at the "B time" $t_{\mathrm{B}}$ be reflected at B in the direction of A, and arrive again at A at the "A time" $t_{\mathrm{A}}^{\prime}$.

In agreement with experience we further assume the quantity

$$
\frac{2 \mathrm{AB}}{t_{\mathrm{A}}^{\prime}-t_{\mathrm{A}}}=c,
$$

to be a universal constant — the velocity of light in empty space." (Einstein, 1923):

In this paper, we distinguish between the former principle as the "principle of constancy of light speed I" and the latter principle as the "principle of constancy of light speed II." The "principle of constancy of light speed I" asserts that the light speed in vacuum does not depend on the speed of the light source. The "principle of constancy of light speed II" asserts that the light speed calculated from the round-trip travel time is constant.

Let there be a given stationary rigid rod of length $L$ as measured by a ruler which is stationary, and assume that the rod is placed along the positive direction of the stationary system's $x$-axis.

Assume that clocks A and B of the same type are set up at points A and B on the rear and front end of this rod. Here clock $A$ will be abbreviated as $C_{A}$, and clock $B$ as $C_{B}$.

Suppose a ray of light is emitted in the direction of $\mathrm{B}$ from $\mathrm{A}$ at time $t_{\mathrm{A}}$ of $\mathrm{C}_{\mathrm{A}}$, reaches and is reflected at $\mathrm{B}$ at time $t_{B}$ of $C_{B}$, and then returns to $A$ at time $t_{\mathrm{A}^{\prime}}$ of $\mathrm{C}_{\mathrm{A}}$. Einstein determined that if the following relationships hold between these times, then the two clocks represent the same time by definition (Einstein, 1923):

$$
\begin{gathered}
t_{\mathrm{B}}-t_{\mathrm{A}}=t_{\mathrm{A}^{\prime}}-t_{\mathrm{B}} . \\
\frac{1}{2}\left(t_{\mathrm{A}}+t_{\mathrm{A}^{\prime}}\right)=t_{\mathrm{B}} .
\end{gathered}
$$

If the relationship in Equation (1) does not hold for the times of $C_{A}$ and $C_{B}$, then it is necessary to adjust the time of $\mathrm{C}_{\mathrm{B}}$ so that the relationship in (1) holds. (Actually, either clock can be adjusted.)

Next, assume that the stationary rod has been accelerated, and has attained the constant velocity $\boldsymbol{v}$ (see Figure 1).

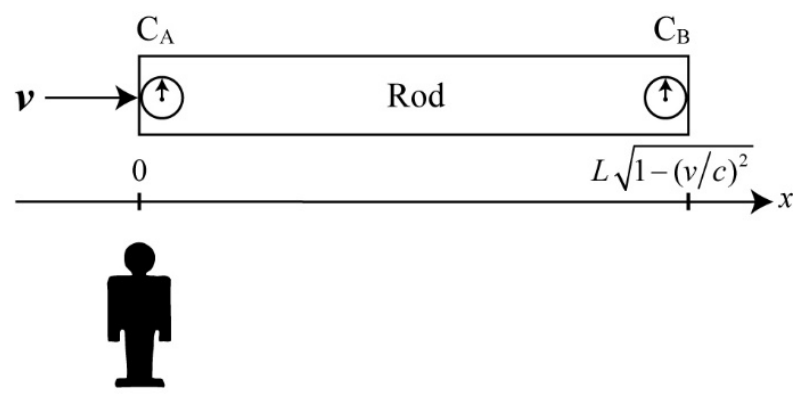

Stationary system

Figure 1 . A rod is moving at constant velocity $v$ relative to stationary system. Clock A and B are set up at A and $\mathrm{B}$ at each end of this rod, and the times of each of these clocks are synchronized while the system is stationary 
Then the time $C_{B}$ must be adjusted again so that the relationship in Equation (1) holds between the times $C_{A}$ and $\mathrm{C}_{\mathrm{B}}$. Due to this operation, the light speed on the outward and return paths measured in the moving system of the rod is measured as $c$ on both paths.

Considered classically, an inertial frame in which light propagates isotropically is a stationary system, and an inertial frame in which light propagates anisotropically is a moving system.

However, if clock time is adjusted according to the requirements of Einstein, light propagates isotropically at the same speed in all inertial frames. (Relativistic isotropic propagation).

Also, all inertial frames become stationary systems in the sense of the theory of relativity.

In this paper, the principle introduced by Einstein is called the "principle of constancy of light speed E." (where "E" stands for Einstein.) That is,

Principle of constancy of light speed E: In all inertial frames, light speed of the outward path and return path is constant $(c)$.

This principle is not a universal principle, but a personal principle introduced by Einstein. To maintain this principle, the observer in a stationary system must adjust the time on a clock each time the velocity of a moving system changes. If the observer neglects this task, the principle of constancy of light speed $\mathrm{E}$ is no longer a principle.

\section{Classical Length Contraction Derived by Applying Principle of Constancy of Light Speed I and II}

Let us imagine that times $t_{\mathrm{A}}, t_{\mathrm{B}}, t_{\mathrm{A}^{\prime}}$ of this moving system corresponds to times $t_{\mathrm{A}}^{\prime}, t_{\mathrm{B}}^{\prime}, t_{\mathrm{A}^{\prime}}^{\prime}$ of the stationary system. Now when the time required for the light signal emitted from point $A$ at the rear of the rod to travel from point $A$ to point $\mathrm{B}$ is measured with the clock in moving system, it is $\left(t_{\mathrm{B}}-t_{\mathrm{A}}\right)$. Also, if this time is measured with the clock in the stationary system, it is expressed as $\left(t_{\mathrm{B}}^{\prime}-t_{\mathrm{A}}^{\prime}\right)$.

According to the STR, the rod seen from stationary system contracts by $1 / \gamma$ times in the direction of motion. Also, the observer in stationary system applies the "principle of constancy of light speed I" to the propagation of light emitted from moving system, and thus $\left(t_{\mathrm{B}}^{\prime}-t_{\mathrm{A}}^{\prime}\right)$ is given by the following equation.

$$
t_{\mathrm{B}}^{\prime}-t_{\mathrm{A}}^{\prime}=\frac{L}{\gamma(c-v)}(\mathrm{s}), \quad \gamma=\left(1-v^{2} / c^{2}\right)^{-1 / 2} .
$$

Also, the time $\left(t_{\mathrm{A}^{\prime}}^{\prime}-t_{\mathrm{B}}^{\prime}\right)$ required for the light signal to return from point $\mathrm{B}$ to point $\mathrm{A}$ is given by the following equation.

$$
t_{\mathrm{A}^{\prime}}^{\prime}-t_{\mathrm{B}}^{\prime}=\frac{L}{\gamma(c+v)}(\mathrm{s})
$$

However, the denominator on the right side of Equations (3) and (4) does not signify that the light speed changes. According to the STR, the relationship of $\left(t_{\mathrm{B}}-t_{\mathrm{A}}\right)$ and $\left(t_{\mathrm{B}}^{\prime}-t_{\mathrm{A}}^{\prime}\right)$ is:

$$
t_{\mathrm{B}}-t_{\mathrm{A}}=\frac{1}{\gamma}\left(t_{\mathrm{B}}^{\prime}-t_{\mathrm{A}}^{\prime}\right)
$$

Here, if the right side of Equation (3) is substituted for $\left(t_{\mathrm{B}}^{\prime}-t_{\mathrm{A}}^{\prime}\right)$ in Equation (5),

$$
t_{\mathrm{B}}-t_{\mathrm{A}}=\frac{L(c+v)}{c^{2}}(\mathrm{~s}) \text {. }
$$

Similarly, if the time $\left(t_{\mathrm{A}^{\prime}}-t_{\mathrm{B}}\right)$ which passes on the clock in moving system while the light signal returns from point $\mathrm{B}$ to point $\mathrm{A}$ is,

$$
t_{\mathrm{A}^{\prime}}-t_{\mathrm{B}}=\frac{L(c-v)}{c^{2}}(\mathrm{~s})
$$

If we set $t_{\mathrm{A}}=0$ to simplify the equation, $t_{\mathrm{A}^{\prime}}$ becomes the time which passes in moving system while the light signal makes a round trip between $A$ and $B$. Thus, the observer in moving system determines that the time of $C_{B}$ when the light has arrived at B is $t_{\mathrm{A}^{\prime}} / 2$. This time can be found from Equations (6) and (7). That is,

$$
\begin{gathered}
\frac{1}{2} t_{\mathrm{A}^{\prime}}=\frac{1}{2}\left[\left(t_{\mathrm{B}}-t_{\mathrm{A}}\right)+\left(t_{\mathrm{A}^{\prime}}-t_{\mathrm{B}}\right)\right] \\
=\frac{L}{c}(\mathrm{~s}) .
\end{gathered}
$$


However, since $L(c+v) / c^{2}>L / c$, the time on $\mathrm{C}_{\mathrm{B}}$ must be later than the time on $\mathrm{C}_{\mathrm{A}}$ to resolve this discrepancy. If this adjustment time is taken to be $\Delta t$,

$$
\begin{array}{r}
\Delta t=\left(t_{\mathrm{B}}-t_{\mathrm{A}}\right)-\frac{1}{2} t_{\mathrm{A}^{\prime}} \\
=\frac{L v}{c^{2}}(\mathrm{~s}) .
\end{array}
$$

If the time of $\mathrm{C}_{\mathrm{B}}$ is delayed by $L v / c^{2}(\mathrm{~s})$, then a state is achieved where the times of $\mathrm{C}_{\mathrm{A}}$ and $\mathrm{C}_{\mathrm{B}}$ can be said to be simultaneous in moving system.

At the time $\Delta t=L v / c^{2}(\mathrm{~s})$, it can be determined that the coordinate system where the rod was initially stationary was the coordinate system where light propagates isotropically.

In this paper, this coordinate system is defined as the classical stationary system $S_{\mathrm{cl}}$. The cl subscript of $S_{\mathrm{cl}}$ is taken to mean a classical inertial frame. Two clocks whose times have been synchronized in $S_{\mathrm{cl}}$ match in an absolute sense.

On the other hand, at the time $\Delta t \neq L v / c^{2}(\mathrm{~s})$, it can be determined that the coordinate system where the rod was initially stationary was the coordinate system where light propagates anisotropically (Suto, 2010):

In this coordinate system, the principle of constancy of light speed II holds, but the principle of constancy of light speed $\mathrm{E}$ does not hold. In this paper, this coordinate system is defined as the classical moving system $S_{\mathrm{cl}}^{\prime}$. The cause of anisotropic propagation of light in $S_{\mathrm{cl}}^{\prime}$ is the velocity vector attached to this coordinate system (Suto, 2015):

The author has previously presented a thought experiment for discriminating between $S_{\mathrm{cl}}$ and $S_{\mathrm{cl}}^{\prime}$. However, Einstein believed it was impossible to discriminate these inertial frames through experiment. Also, Einstein proposed that the time on two clocks in an inertial frame be adjusted so that the relationship in Equation (1) holds. As a result, the speed of light became $c$ for both the outward path and return path, even in $S_{\mathrm{cl}}^{\prime}$. Also, all inertial frames became equivalent in the sense of the theory of relativity (a stationary system $S_{\mathrm{re}}$ in the sense of the theory of relativity). The following summarizes the above:

$$
\text { Classical physics Special theory of relativity }
$$

$$
\left\{\begin{array}{l}
\text { Classical stationary system } S_{\mathrm{cl}} \\
\text { Classical moving system } S_{\mathrm{cl}}^{\prime}
\end{array}\right\} \rightarrow \text { Relativistic stationary system } S_{\mathrm{re}}
$$

Now, how should we imagine $S_{\mathrm{cl}}$ ? In the latter half of the 19th century, it was thought that a medium was needed for light to propagate as a wave. The physicists at the time called this medium the "aether." However, Einstein eliminated the aether from the STR, and thus discussion of the existence of this hypothetical substance gradually disappeared. However, if the principle of constancy of light speed I holds, then there needs to be a medium for transmitting light as a wave. Thus, this paper looks at the pairs of virtual particles and antiparticles which constitute the vacuum. The countless relative velocities between the $S_{\mathrm{cl}}$ coordinate system and the countless virtual particle pairs in the vicinity are indicated as vectors, and then composed. If the size of the vector becomes zero at this time, then the coordinate system is $S_{\mathrm{cl}}$ where light propagates isotropically. On the other hand, if the composed vector has magnitude, then the coordinate system is $S_{\mathrm{cl}}^{\prime}$ where light propagates anisotropically.

\section{Length Contraction and Time Delay Explainable using Classical Considerations}

Consider a laboratory whose interior floor is a square. The Michelson interferometer is placed in this laboratory (see Figure 2). At the center of the room, there is a glass plate (beam splitter) $\mathrm{P}$ with a semi-transparent metal coating on its front face. The angle between this glass plate and the $x$-axis is $45^{\circ}$. Light emitted from the light source $\mathrm{S}$ strikes this glass at an angle, and the light is split in two. One beam passes through the plate, strikes a mirror $\mathrm{M}_{x}$, is reflected, and retraces its path to the splitting point P. On the second light path, the beam is reflected by the glass plate $\mathrm{P}$, arrives at mirror $\mathrm{M}_{y}$, is reflected there, and returns to the splitting point $\mathrm{P}$. (Only the essential parts of the experimental instrument are shown here. Equipment not needed for the discussion in this paper has been omitted.)

This laboratory is moving at constant velocity $\boldsymbol{v}$ along the $x$-axis of $S_{\mathrm{cl}}$. The light path length $\mathrm{PM}_{x}$ measured indoors is taken to be $L_{x}$ and the path length $\mathrm{PM}_{y}$ is taken to be $L_{y}$. (However, in measurements in the laboratory, $L_{x}$ and $L_{y}$ are equal.) In addition, the light path length when $L_{x}$ is measured from $S_{\mathrm{cl}}$ is taken to be $L_{x}^{\prime}$, and the light path length when $L_{y}$ is measured from $S_{\mathrm{cl}}$ is taken to be $L_{y}^{\prime}$. (However, $L_{y}$ and $L_{y}^{\prime}$ are equal.) 


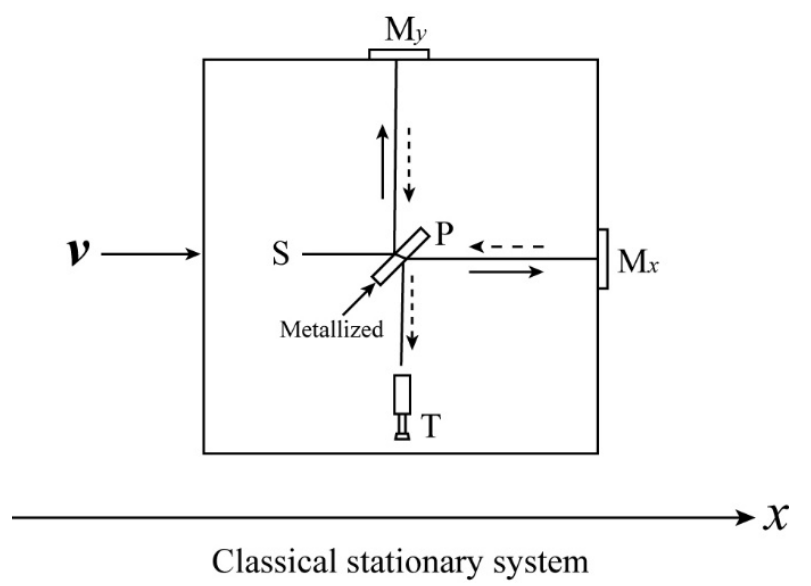

Figure 2. This figure shows the view from above of a laboratory moving at constant velocity with respect to $S_{\mathrm{cl}}$

Here, the time required for light to make a round trip over $\mathrm{PM}_{x}$ is measured from $S_{\mathrm{cl}}$. If this round trip time is taken to be $t_{x}^{\prime}$, then the observer in $S_{\mathrm{cl}}$ applies the principle of constancy of light speed I to this light propagation, and thus:

$$
t_{x}^{\prime}=\frac{L_{x}^{\prime}}{c-v}+\frac{L_{x}^{\prime}}{c+v}=\frac{2 L_{x}^{\prime} c}{c^{2}-v^{2}}=\frac{2 L_{x}^{\prime}}{c\left(1-v^{2} / c^{2}\right)} .
$$

Next, the time for light to make a round trip over $\mathrm{PM}_{y}$ is measured. If this round trip time is measured in $S_{\mathrm{cl}}$ and taken to be $t_{y}^{\prime}$, then:

$$
t_{y}^{\prime}=\frac{2 L_{y}^{\prime}}{c\left(1-v^{2} / c^{2}\right)^{1 / 2}} .
$$

The method of deriving Equation (11) is explained in many textbooks so here it is omitted (Feynman, 1963; French, 1968):

Incidentally, the predicted effect could not be detected from the Michelson-Morley experiment. This means that $t_{x}^{\prime}$ and $t_{y}^{\prime}$ are equal. In the end, the following relationship can be derived from Equations (10) and (11).

$$
L_{x}^{\prime}=\frac{L_{y}^{\prime}}{\gamma}
$$

Here, $L_{y}^{\prime}$ and $L_{x}$ are equal, so Equation (12) can be written as follows.

$$
L_{x}^{\prime}=\frac{L_{x}}{\gamma}
$$

When measured from $S_{\mathrm{cl}}$, the laboratory contracts by $1 / \gamma$ times in the direction of motion. This contraction is physical contraction due to the fact that some force has acted on the laboratory, and this can be regarded as true contraction (contraction I).

Incidentally, an observer in the coordinate system $S_{\mathrm{cl}}^{\prime}$ of the laboratory applies the principle of constancy of light speed II to this light propagation, and thus the round trip times of light $t_{x}$ and $t_{y}$ are predicted as follows:

$$
\begin{aligned}
t_{x} & =\frac{2 L_{x}}{c} . \\
t_{y} & =\frac{2 L_{y}}{c} .
\end{aligned}
$$

In the end, $t_{y}^{\prime}$ elapses in $S_{\mathrm{cl}}$ while $t_{y}$ elapses in $S_{\mathrm{cl}}^{\prime}$. In addition, $L_{y}=L_{y}^{\prime}$ and thus Equation (11) can be written as follows:

$$
t_{y}^{\prime}=\frac{2 \gamma L_{y}}{c} .
$$


Next, if this is compared with Equations (15) and (16):

$$
t_{y}^{\prime}=\gamma t_{y}
$$

When observed from $S_{\mathrm{cl}}$, the time which elapses in $S_{\mathrm{cl}}^{\prime}$ is delayed compared to the time which elapses in $S_{\mathrm{cl}}$. Actually, this prediction has been verified by experiments where the life of elementary particles is extended. In the end, space contraction and time delay in $S_{\mathrm{cl}}^{\prime}$ can be predicted if the principles of constancy of light speed I and II are assumed.

\section{Rod Contraction which can and cannot be Classically Explained}

In this section, the lengths of rod A (inertial frame A) and B (inertial frame B) moving at constant velocity relative to each other are measured using two types of methods.

1) Two methods for an observer in inertial frame $\mathrm{A}\left(S_{\mathrm{cl}}\right)$ to measure the length of rod B moving at constant velocity

Measurement 1. In this case, observer $A_{1}$ is at the rear end and observer $A_{2}$ is at the front end of rod $A$ of length $L$ placed on the $x$-axis of $S_{\mathrm{cl}}$. Also, at an arbitrary time, a light signal is emitted from a point light source $\mathrm{S}$ placed in the center of rod $\mathrm{A}$. That light signal propagates isotropically from $\mathrm{S}$, and arrives at both ends of the rod with absolute simultaneity. At this time, observers $\mathrm{A}_{1}$ and $\mathrm{A}_{2}$ read off the position of both ends of rod A from the $x_{\mathrm{B}}^{\prime}$ coordinates. (This $x_{\mathrm{B}}^{\prime}$-axis is parallel to the $x$-axis.)

Since rod B contracts by $1 / \gamma$ times in the direction of motion, the length of rod A read off from the $x_{B}^{\prime}$-axis becomes $\gamma L$ if we refer to Equation (15). From this,

$$
\text { Length of rod A : Length of rod B, } \quad \gamma L: L \rightarrow 1: \frac{1}{\gamma}, \quad \gamma>1 .
$$

Here, if the length of rod B measured from $S_{\mathrm{cl}}$ is taken to be $L^{\prime}$, then Equation (18) can be written as follows;

$$
L^{\prime}=\frac{L}{\gamma}
$$

Contraction in this case is a result of the fact that some physical force acted on rod B, and this can be called true contraction (contraction I).

Measurement 2. First we consider rod B moving at constant velocity $v$ along the $x$-axis of $S_{\mathrm{cl}}$. (Length when the rod is at rest is $L$.) When the front end of the rod passes in front of observer A in $S_{\mathrm{cl}}$, observer A starts the stopwatch, and measures the time $t_{\mathrm{A}}$ until the rear end of the rod passes. According to the STR, the rod B contracts by $1 / \gamma$ times in the direction of motion at this time. That is,

$$
L^{\prime}=v t_{\mathrm{A}}=\frac{L}{\gamma} .
$$

The results obtained from measurement 1 and 2 verify the contraction in Equation (13).

2) Two methods for an observer in inertial frame $\mathrm{B}\left(S_{\mathrm{cl}}^{\prime}\right)$ to measure the length of rod $\mathrm{A}$.

Measurement 3a. In this case, contrary to measurement 1, observers at both ends of rod B compare the length of rod B and A with absolute simultaneity. The clocks are used at both ends of rod B have been synchronized when the rod was at rest in $S_{\mathrm{cl}}$. If Equation (19) is taken into account, the length of rod B read off from the $x$ coordinates by observers at both ends of rod B is shorter than rod A. That is,

$$
\text { Length of rod B : Length of rod A, } \frac{L}{\gamma}: L \rightarrow 1: \gamma .
$$

Considered classically, if rod B contracts, then rod A is longer than rod B.

Measurement $4 \mathrm{a}$. This case is the inverse of measurement method 2. Observer on rod B measures the length of rod A of length $L$ placed on the $x$-axis of $S_{\mathrm{cl}}$. If observer measures the time required to pass both ends of rod A, and this is taken to be $t_{\mathrm{B}}$, then classically $t_{\mathrm{B}}$ is,

$$
t_{\mathrm{B}}=\frac{L}{v} \text {. }
$$

However, the time which passes in the coordinate system of rod B is delayed compared to the time which passes in $S_{\mathrm{cl}}$. Therefore, the time $t_{\mathrm{B}}$ which passes in $S_{\mathrm{cl}}^{\prime}$ becomes $1 / \gamma$ times Equation (22). That is, 


$$
t_{\mathrm{B}}=\frac{L}{\gamma v} .
$$

Incidentally, it is impossible for rod A to contract because rod B began to move at constant velocity. Thus, the observer of rod B determines that time elapsing in his own coordinate system is delayed, and he does not regard rod A as having contracted. In classical measurement, contraction of rod A cannot be observed.

\section{Contraction of Rod Interpreted by Borrowing Einstein's Measurement Method}

The measurement in this section employs the following operation and principle used when developing the STR.

1) Times on the clocks at both ends of rod B moving at constant velocity are synchronized so that the relationship in Equation (1) holds.

2) The principle of relativity is applied to the coordinate system of rod B.

Measurement $3 b$. Next, the moving observer B uses the same method as measurement method 1, and reads off the position of both ends of rod B from the $x$ coordinate in $S_{\mathrm{cl}}$. Observer $\mathrm{B}_{1}$ is at the rear end and observer $\mathrm{B}_{2}$ is at the front end of the moving rod $\mathrm{B}$.

At an arbitrary time, a light signal is emitted from $\mathrm{S}_{\mathrm{B}}$ in the center of rod B. An observer in $S_{\mathrm{cl}}$ applies the principle of constancy of light speed I to this light propagation. When the light signal emitted from $\mathrm{S}_{\mathrm{B}}$ has arrived at both ends of the rod, observers $\mathrm{B}_{1}$ and $\mathrm{B}_{2}$ read off the $x$ coordinates in $S_{\mathrm{cl}}$.

Then the two observers of rod B compare the length of the $x$ coordinate they themselves read off, and the length of the stationary $\operatorname{rod} \mathrm{A}$.

Now, the observer in $S_{\mathrm{cl}}$ measures time until the light signals emitted from $\mathrm{S}_{\mathrm{B}}$ arrives at the observers $\mathrm{B}_{2}$ and $\mathrm{B}_{1}$ at both ends of the rod. If these times are taken to be $t_{2}^{\prime}$ and $t_{1}^{\prime}$, then since the distance from $\mathrm{S}_{\mathrm{B}}$ to the rod end is $\operatorname{rod} L / 2$,

$$
\begin{aligned}
& t_{1}^{\prime}=\frac{L}{2 \gamma(c+v)} . \\
& t_{2}^{\prime}=\frac{L}{2 \gamma(c-v)} .
\end{aligned}
$$

Incidentally, the observer in $S_{\mathrm{cl}}$ determines the following values for the distance traveled by the light signal until it reaches both ends of the rod.

Travel distance $x_{-}$in the negative direction of the $x$-axis

$$
x_{-}=c t_{1}^{\prime}=\frac{L c}{2 \gamma(c+v)} .
$$

Travel distance $x_{+}$in the positive direction of the $x$-axis

$$
x_{+}=c t_{2}^{\prime}=\frac{L c}{2 \gamma(c-v)} .
$$

The observers at both ends of rod B obtain the following values as the length of the rod read off from the $x$-axis of the stationary system, based on Equations (26) and (27).

$$
L^{\prime}=x_{+}+x_{-}=\gamma L, \quad L<L^{\prime} .
$$

Contrary to Equation (21), the length of rod B in this case is longer than rod A. That is,

$$
\text { Length of } \operatorname{rod} \mathrm{B}: \text { Length of } \operatorname{rod} \mathrm{A}, \quad \gamma L: L \rightarrow \gamma: 1 .
$$

Incidentally, if the principle of relativity is applied to the coordinate system of rod $\mathrm{B}$, the length of A must match Equation (19).

Thus, the observers on rod B make the following judgment based on Equation (28).

$$
\gamma L: L \rightarrow 1: \frac{1}{\gamma} .
$$

When the length of $\operatorname{rod} \mathrm{A}$ is measured from the coordinate system of $\operatorname{rod} \mathrm{B}, \operatorname{rod} \mathrm{A}$ is contracted by $1 / \gamma$ times in the direction of motion (contraction II). 
Measurement $4 \mathrm{~b}$. An observer of rod B who has applied the principle of relativity believes that his own coordinate system is a stationary system. Therefore, Equation (23) is explained using the contraction of rod A rather than a delay in the time of $S_{\mathrm{cl}}^{\prime}$ (contraction III). If it is assumed that the principle of relativity holds in $S_{\mathrm{cl}}^{\prime}$, then the results of measurement $3 \mathrm{~b}$ and $4 \mathrm{~b}$ match the values of measurement 1 and 2 .

\section{Discussion}

In section 4, an observer in $S_{\mathrm{cl}}$ applied the principle of constancy of light speed I, and an observer in $S_{\mathrm{cl}}^{\prime}$ applied the principle of constancy of light speed II, to propagation of light emitted from a light source in $S_{\mathrm{cl}}^{\prime}$. At this time, the length of the laboratory measured by the observer in $S_{\mathrm{cl}}$ contracted in the direction of motion. This contraction is physical contraction which occurred as a result of some force having acted on the moving laboratory, and is true contraction (contraction I).

Next, in measurement 3a, clocks synchronized in $S_{\mathrm{cl}}$ were used as the two clocks in $S_{\mathrm{cl}}^{\prime}$ used for measurement. Therefore, the times of the two clocks matched absolutely. The observer in $S_{\mathrm{cl}}^{\prime}$ determined the rod B to be shorter than rod A (Equation (21)).

Thus, the method of clock synchronization proposed by Einstein was used in this paper. As a result, in measurement 3b, rod B was determined to be longer than rod A (Equation (29)).

However, even this is unacceptable, and therefore in this case the principle of relativity was applied to this coordinate system. According to the principle of relativity, any two inertial frames are equivalent, and thus measured values must match. In measurement $3 \mathrm{~b}$, the ratio of the lengths of $\operatorname{rod} \mathrm{B}$ and $\operatorname{rod} \mathrm{A}$ was interpreted as $1: 1 / \gamma$ (contraction II). However, actually it is not the case that rod A has physically contracted. This is relativistic contraction which occurs when measurement is done using the method indicated by Einstein.

The delay in time which elapses in the coordinate system of rod B was observed in measurement $4 \mathrm{a}$, but it was not determined that rod A contracted based on Equation (23). However, in measurement $4 \mathrm{~b}$ the principle of relativity was applied to inertial frame B, and therefore, observer B believed his own coordinate system to be a stationary system. With regard to the fact that Equation (23) was obtained, it was determined to be the result of rod A having contracted. This contraction is tentative contraction (contraction III) observed because the passage of time in inertial frame B was delayed. With this, the values for measurements 1 to 4 all match.

Now, how is this problem handled in the STR? The STR assumes the principle of relativity. If contraction of rod $\mathrm{B}$ is observed in measurement 1 and 2, then by definition contraction of rod $\mathrm{A}$ is also observed in measurement $3 \mathrm{~b}$ and $4 \mathrm{~b}$. If physics is a science which pursues the nature of reality as it is, then contraction II and contraction III cannot be accepted. In the end, the STR should be regarded not as something which describes physical law existing in the natural world, but as a mathematical expression of the universe as imagined by Einstein. The velocity vectors present in the natural world are missing from the STR.

\section{Conclusion}

Through the discussion in this paper, it was determined that there are the following three types of contraction of a rod moving at constant velocity.

Contraction I (physical contraction): This is the contraction obtained from measurements 1 and 2, and it is true contraction due to fact that some physical force has acted on rod B which is moving at constant velocity. It was possible to explain this contraction with the classical discussion in section 5 .

Contraction II (relativistic contraction): Reasons why contraction of rod A was observed in measurement $3 \mathrm{~b}$ :

1) True contraction of rod B which is moving at constant velocity (contraction I)

2) Times of the clocks at both ends of rod B were adjusted to achieve simultaneity in the sense of the theory of relativity.

3) The principle of relativity was applied to inertial frame B.

It was possible to predict Equation (29) from 1) and 2). In addition, by applying the principle of relativity, it was possible to interpret Equation (29) like Equation (30), and explain the contraction of rod A.

Contraction III (relativistic contraction): The principle of relativity was applied to inertial frame B in measurement $4 \mathrm{~b}$. Therefore, with regard to the fact that Equation (23) was obtained, it was determined to be the result of rod A having contracted.

In measurement 1 and $3 \mathrm{~b}$, there was contraction I and II so results matching the predictions of the STR were obtained. Also, in measurement 2 and 4b, contraction I and III are the reason why symmetry of length contraction was observed. 
In the end, the fact that symmetry of rod contraction, which is classically impossible, could be explained in this paper is due to the following three reasons.

1) The inertial frame A was assumed to be a classical stationary system.

2) An operational definition of simultaneity was used in measurement $3 b$.

3) In measurement $3 b$ and measurement $4 b$, the principle of relativity was applied to inertial frame $B$.

As a result, relativistic contraction II and III occurred, and the measurement results in measurement $3 \mathrm{~b}$ and measurement $4 \mathrm{~b}$ matched the results of measurement 1 and measurement 2 .

The STR is an astonishing theory in which rod A, undergoing no change in itself, is forced to contract. This paper concludes that there should be serious discussion of whether or not the STR can really be called a physical theory.

\section{Acknowledgments}

I would like to express my thanks to the staff at ACN Translation Services for their translation assistance. Also, I wish to express my gratitude to Mr. H. Shimada for drawing figures.

\section{References}

Einstein, A. (1923). The Principle of Relativity (pp. 40-41). New York: Dover Publication, Inc.

Feynman, R. P. (1963). The Feynman Lectures on Physics (pp. 15-4). Addison-Wesley Publishing Company.

French, A. P. (1968). Special Relativity (p. 55). New York \& London: W.W.NORTON\&COMPANY.

Suto, K. (2010). Violation of the special theory of relativity as proven by synchronization of clocks. Physics Essays, 23(3), 511-519. http://dx.doi.org/10.4006/1.3474836

Suto, K. (2015). Demonstration of the existence of a velocity vector missing from the special theory of relativity. Physics Essays, 28(3), 345-351. http://dx.doi.org/10.4006/0836-1398-28.3.345

Suto, K. (2016). Thought Experiment Revealing a Contradiction in the Special Theory of Relativity. Applied Physics Research, 8(6), 70-74. http://dx.doi.org/10.5539/apr.v8n6p70

Suto, K. (2016). Elucidation of Time Symmetry Predicted by the Special Theory of Relativity. IOSR Journal of Applied Physics, 8(6) IV, 20-27. http://dx.doi.org/10.9790/4861-0806042027

Suto, K. (2017). A New Problem with the Twin Paradox. Applied Physics Research, 9(2), 77-86 https://doi.org/10. 5539/apr.v9n2pxx

\section{Copyrights}

Copyright for this article is retained by the author(s), with first publication rights granted to the journal.

This is an open-access article distributed under the terms and conditions of the Creative Commons Attribution license (http://creativecommons.org/licenses/by/4.0/). 